\title{
Musical Auditory Hallucinosis from Listeria Rhombencephalitis
}

\author{
Andre G. Douen and Pierre R. Bourque
}

\begin{abstract}
Background: Complex auditory hallucinations have rarely been reported in cases of brainstem stroke or tumor. Method: Case study. Results: A patient with acute Listeria rhombencephalitis complained of formed musical auditory hallucinations on the side of recent sensorineural deafness. MRI revealed an abscess in the middle cerebellar peduncule with extensive surrounding edema. Conclusions: Disruption of brainstem auditory pathways may cause complex auditory hallucinations. Potential pathogenetic mechanisms are discussed and a diagnostic approach is proposed.
\end{abstract}

\begin{abstract}
RÉSUMÉ: Hallucinose auditive musicale due au Listeria rhombencephalitis. Introduction: Des hallucinations auditives complexes ont rarement été rapportées dans les cas d'accidents vasculaires cérébraux ou de tumeurs du tronc cérébral. Méthode: Observation clinique. Résultats: Un patient porteur d'une infection aiguè à Listeria rhombencephalitis s'est plaint d'hallucinations auditives musicales du côté d'une surdité de perception d'apparition récente. La RMN a monté un abcès dans le pédoncule cérébral moyen avec un oedème environnant important. Conclusions: La perturbation des voies auditives au niveau du tronc cérébral peut causer des hallucinations auditives complexes. Nous discutons des mécanismes pathogéniques potentiels et nous proposons une approche diagnostique.
\end{abstract}

Can. J. Neurol. Sci. 1997; 24: 70-72

Complex auditory hallucinations ( $\mathrm{AH})$ are frequently a symptom of psychiatric disease. They have also been described with lesions of the peripheral auditory pathways ${ }^{1.6}$ or cerebral hemisphere. ${ }^{5-9}$ The pathophysiology of complex AH remains controversial. Their occurrence with peripheral auditory lesions suggests a role for deafferentation in the central release of auditory memories. ${ }^{1.5 .10 .11}$ In contrast, a direct role of the cerebrum is suggested by the association of AH with brain tumors, epilepsy and stroke, in the absence of any hearing loss. ${ }^{5,6}$

Recently, cases of AH due to brainstem stroke, haemorrhagic and ischemic, have been described. ${ }^{11-14}$ We report an association with Listeria rhombencephalitis, which to our knowledge has not been previously described. The possible mechanism of such brainstem $\mathrm{AH}$ will be discussed and an approach to the diagnosis of new onset $\mathrm{AH}$ is proposed.

\section{Case Report}

A 43-year-old man presented with a prodrome of bifrontal headache, fever, nausea, vomiting and a three day history of left sided perioral paraesthesia. At the time of admission he also complained of difficulty with balance and taste. He had a past history of hypertension, hypercholesterolemia, obstructive sleep apnoea and alcohol abuse.

On admission, his temperature was $38.7^{\circ} \mathrm{C}$. There was no lymphadenopathy nor nuchal rigidity. The positive findings on the neurologic examination were: a) right-beating jerk nystagmus in the primary position of gaze, exacerbated upon looking to the right; b) decreased pain sensation in the left trigeminal territory; c) mild gait ataxia with a tendency to fall backwards on Romberg testing. The initial CT scan of the brain was normal. Lumbar puncture revealed a clear CSF with cell counts of $7 \mathrm{RBC} / \mathrm{mm}^{3}$ and $143 \mathrm{WBC} / \mathrm{mm}^{3}$ (42\% polymorphonuclear cells). The CSF to serum glucose ratio was $50 \%$. The CSF protein level was $0.92 \mathrm{~g} / \mathrm{L}$. Listeria monocytogenes was identified in blood cultures.

Despite immediate treatment with cefotaxime $1 \mathrm{~g}$ IV Q8H and ampi- cillin $2 \mathrm{~g}$ IV Q6H, his neurological condition deteriorated in the 48 hours following admission. He developed progressive deficits involving the left cranial nerves V, VII, VIII, IX and X, as well as left-sided limb ataxia. Hearing acuity was markedly reduced on the left side and the Weber test lateralized to the right. He complained of nearly constant musical auditory hallucinations originating from his deaf ear. These consisted of orchestral melodies ("1950's big band music, mostly Miller") although at other times specific songs with words such as Summer Girl, were recognized. His sensorium remained clear. He felt that the hallucinations were odd, at times a mild nuisance, at times a somewhat pleasant distraction.

Serial CT scans showed an evolving abscess immediately lateral to the left side of the fourth ventricle. MRI with gadolinium enhancement, obtained eight days after admission, showed a $1.8 \mathrm{~cm}$ diameter ringenhancing lesion in the left middle cerebellar peduncle (Figure 1a). There was no meningeal enhancement. Unenhanced T2 weighted images demonstrated marked surrounding edema in the adjacent cerebellum and in the left side of the pons and medulla (Figure 1b).

The clinical course was further complicated by haemorrhage in the area of rhombencephalitis and this was surgically evacuated on the tenth hospital day. There was gradual improvement thereafter, although he was left with mild hemiparesis and multiple partial cranial neuropathies. Auditory hallucinations gradually subsided over a period of one month. Their disappearance coincided with the return of hearing in the left ear. Audiometry was only done at the time of recovery. It revealed a mild impairment of speech discrimination on the left with moderate high frequency bilateral sensorineural hearing loss.

From the Division of Neuroscience, Ottawa Civic Hospital, University of Ottawa, Ottawa.

RECEIVED MAY 13, 1996. ACCEPTED IN FINAL FORM JULY 18, 1996

Reprint requests to: Dr. Pierre R. Bourque, Ottawa Civic Hospital, Section D7, 1053 Carling Avenue, Ottawa, Ontario, Canada K1Y 4E9 


\section{Discussion}

Listeria rhombencephalitis is a rare infection with a high mortality and frequent neurologic sequelae in survivors. ${ }^{15}$ As part of his acute presentation, this patient developed $\mathrm{AH}$ and severe unilateral sensorineural deafness. The definite musical and verbal content of his auditory experiences, as well as his preserved insight and clear sensorium establish that these are hallucinations, not auditory illusions nor simple tinnitus. Neuroimaging and clinical findings allow inferences as to the likely site of pathology. MRI showed an abscess in the middle cerebellar peduncle, with extensive edema in the neighbouring medulla and pons. Clinical deficits implicating cranial nerves 5 , 7,9 and 10 , with sparing of the abducens nerve, medial lemniscus and corticospinal tract would suggest involvement of the dorsolateral pontomedullary junction. Although extraaxial involvement of these cranial nerves by meningitis cannot be completely excluded, such unilateral clustering would be very unlikely and meningeal hyperintensity was not seen on the gadolinium-enhanced MRI study. In addition, there was no clinical, MRI, nor electroencephalographic evidence of supratentorial involvement. Thus the central auditory structures most likely to be implicated in this case are the left cochlear nuclei, acoustic striae, superior olive, trapezoid body and caudal lateral lemniscus. There is bilateral representation in the auditory pathways ascending from the cochlear nuclei, such that lesions of the lateral lemniscus or more rostral auditory structures cause only partial bilateral hearing loss. The profound left-sided deafness noted in our patient suggests significant involvement of the ipsilateral cochlear nuclei.

Brainstem AH have been reported previously in five cases of pontine haemorrhage, ${ }^{1-14}$ four cases of pontine tegmental infarction $^{14}$ and one case of metastasis to the midbrain. ${ }^{13}$ The anatomical structures implicated ranged in caudal-rostral extent

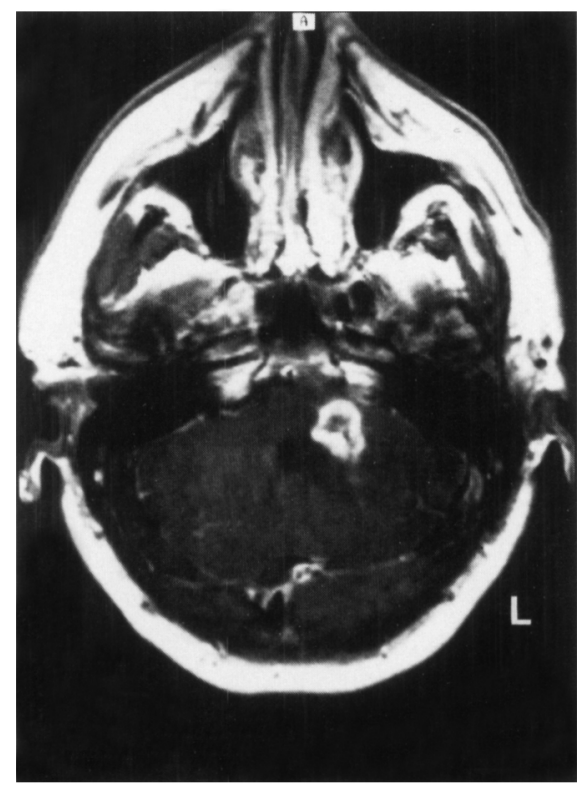

Figure Ia: T1-weighted axial MRI following infusion of gadolinium. There is a ring-enhancing oval abscess in the left middle cerebellar peduncle. (TR 600ms, TE 20ms).

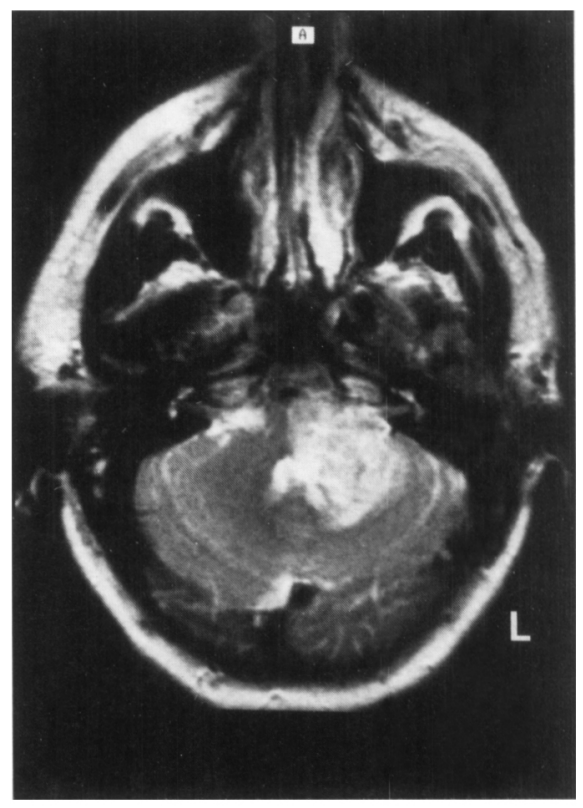

Figure 1b: T2-weighted axial MRI showing extensive hyperintensity in keeping with edema of the left half of the lower pons and adjacent cerebellar hemisphere. (TR 2000ms, TE $90 \mathrm{~ms}$ ).

from the cochlear nuclei to the inferior colliculi. Serial neuropathological sections of the brainstem were examined only in the case of Cascino and Adams and revealed destruction of the inferior colliculus and adjacent lateral lemniscus, with sparing of more caudal auditory pathways. ${ }^{13}$ On the basis of clinical, MRI and brainstem evoked potential data, Lanska and Murata have postulated involvement of more caudal structures, namely the acoustic striae, trapezoid body and superior olivary nucleus, ${ }^{11,12}$ consistent with the localization observed in the present case.

In our patient, as in all previously reported cases of brainstem $\mathrm{AH}$, hearing loss occurred ipsilateral to the ear in which hallucinations were perceived to arise. Elementary and musical hallucinations have been extensively described in cases of acquired chronic deafness of peripheral etiology. ${ }^{1-6.16,17}$ In this context, hallucinations tend to occur after years of sensorineural or conductive hearing loss and run a fluctuating course. They generally begin insidiously, taking the form of elementary tinnitus of variable pitch or more complex, engine-like sounds. Gordon has argued that most, if not all, $\mathrm{AH}$ have a peripheral generator. ${ }^{3}$ Indeed, individual hair cells of the bullfrog sacculus have been implicated in the production of cochlear distortions, ${ }^{18}$ although there is no evidence that this mechanism would lead to more complex AH. Clearly, AH which include verbal content or recognition of a specific melodic line must involve activation of cerebral cortical areas.

In the present case, complex $\mathrm{AH}$ appeared within a few days of an acute brainstem injury. Their disappearance was concurrent with neurologic improvement, further suggesting an etiologic link. Conceivably, in $\mathrm{AH}$ of peripheral or brainstem origin, the release of auditory memories is triggered by auditory input deprivation. ${ }^{11-14}$ The pathways mediating such 
selective cortical disinhibition or hyperexcitability are unknown. Hypnagogic hallucinations, which may feature visual, auditory or somaesthetic perceptions, may represent a useful model. They consist of a dissociation of dream-like experiences from their usual substrate, sleep. Pontine neurons responsible for ponto-geniculo-occipital waves (which also project to parietal and temporal association areas), may be the generators of hypnagogic hallucinations. ${ }^{19}$ These neurons appear to be inhibited by pontine raphe neurons, which have been a site of pathology in many reported cases of brainstem AH. Peduncular hallucinosis also bears similarity to brainstem $\mathrm{AH}$, although here vesperal visual hallucinations are the hallmark and the pathology is mesencephalic rather than predominantly pontine.

Complex AH may be a source of great patient anxiety and often pose a diagnostic challenge. When there is a definite verbal content, a psychiatric disorder can be suspected in the context of a normal neurologic examination, prior history of psychiatric disease, impaired insight and association with other psychotic symptoms. The presence of chronic or new hearing loss should be assessed, as auditory deprivation may play an important role in AH. Although rare, focal supratentorial and brainstem pathology should also be considered. This report and others emphasize an association of complex $\mathrm{AH}$ with neoplastic, vascular or inflammatory disorders of the brainstem, particularly the pontine tegmentum. In all cases reported so far the brainstem localization was evident from the clinical examination. Huwever, it is conceivable that a more limited structural brainstem lesion could cause selective auditory pathway deafferentation. Consequently, focal infratentorial pathology should be considered in the assessment of patients with complex $\mathrm{AH}$.

\section{REFERENCES}

1. Hammeke TA, McQuillen MP, Cohen BA. Musical hallucinations associated with acquired deafness. J Neurol Neurosurg Psychiatry 1989; 46: 570-572.

2. Wengle SP, Burke WJ, Holeman D. Musical hallucinations; the sounds of silence? Am Geriatr Soc 1989; 37: 163-166.

3. Gordon AG. Musical hallucinations (letter). Neurology 1994; 44: 986.

4. Gilbert CJ. Pentoxifylline-induced musical hallucinations (letter). Neurology 1993; 43: 1621-1622.

5. Berrios GE. Musical hallucinations: a historical and clinical study. $\mathrm{Br}$ J Psychiatry 1990; 156: 188-194.

6. Berrios GE. Musical hallucinations: a statistical analysis of 46 cases. Psychopathology 1991; 24: 356-360.

7. Paquier $P$, Van Vugt $P, B a l ~ P$, et al. Transient musical hallucinosis of central origin: a review and clinical study. J Neurol Neurosurg Psychiatry, 1992; 55: 1069-1073.

8. Wordaz N, Becker T, Cockert J. Musical hallucinations associated with post-thyroidectomy hypoparathyroidism and symmetric basal ganglia calcifications (letter). J Neurol Neurosurg Psychiatry 1995; 58: 763-764.

9. Fenelon G, Marie S, Ferrior J-P, Guillard A. Hallucinose musicale: 7 cas. Rev Neurol 1993; 149 (8-9): 462-467.

10. Inzelberg R, Vishnievskaya S, Korezyn A. Transient musical hallucinosis. J Neurol Neurosurg Psychiatry 1993; $56: 833$

11. Lanska DJ, Lanska MJ, Mendez M. Brainstem auditory hallucinosis. Neurology 1987; 37: 1685.

12. Murata S, Naritomi H, Sawada T. Musical auditory hallucinations caused by a brainstem lesion. Neurology 1994; 44: 156-158.

13. Cascino GD, Adams RD. Brainstem auditory hallucinosis. Neurology 1986; 36: 1042-1047.

14. Cambier J, Decroix JP, Masson C. Hallucinose auditive dans les lésions du tronc cérébral. Rev Neurol 1987; 143: 255-262.

15. Armstrong RW, Fung PC. Brainstem encephalitis (rhombencephalitis) due to listeria monocytogenes: case report and review. Clin Infect Dis 1993; 16: 689-702.

16. Freeland A, O'Reilly R. Musical hallucinations: another case report (letter). Can J Psychiatry 1992; 37: 731.

17. Fishman M. Musical hallucinations: report of two unusual cases. Can J Psychiatry 1991; 36: 609-611.

18. Jaramillo F, Markin VS, Hudspeth AJ. Auditory illusions and the single hair cell. Nature 364: 527-529.

19. Mitler MM. Toward an animal model of narcolepsy-cataplexy. In: Guillemineault C, Dement WC and Passouant P, eds. Narcolepsy Spectrum Publ. New York, 1976: 384-409. 